\title{
INVESTIGANDO EM $C$ : UMA UNIDADE DE APRENDIZAGEM ONLINE PARA ESTUDO DE NÚMEROS COMPLEXOS
}

\author{
Silvia Cristina Freitas Batista, PGIE/UFRGS, IF Fluminense Campus Campos-Centro \\ silviac@iff.edu.br \\ Gilmara Teixeira Barcelos, PGIE/UFRGS, IF Fluminense Campus Campos-Centro \\ gilmarab@iff.edu.br \\ Débora Maciel da Costa, IF Fluminense Campus Campos-Centro \\ debbymcosta@gmail.com \\ Patricia Alejandra Behar, NUTED-PGIE/UFRGS \\ patricia.behar@ufrgs.br
}

\section{RESUMO}

Os recursos digitais abrem importantes possibilidades para o processo de ensino e aprendizagem de Matemática, tais como simulação, visualização, experimentação, entre outras. Nesse sentido, descreve-se o desenvolvimento da unidade de aprendizagem online "Investigando em $C$ ", direcionada ao estudo de Números Complexos, no Ensino Médio. A unidade foi desenvolvida em HTML e contém, entre outros recursos, 13 applets elaborados com o objetivo de possibilitar o estudo de Números Complexos, de forma associada à Geometria Analítica, e uma apostila de atividades investigativas relacionadas aos applets. A unidade foi submetida a uma validação preliminar realizada com professores e licenciandos em Matemática, por meio de um minicurso. Descreve-se a referida validação e analisam-se os resultados obtidos. Estes mostraram que a unidade está adequada ao seu objetivo, ou seja, pode colaborar para o estudo de Números Complexos, sob uma perspectiva menos algébrica.

Palavras-chave: Investigando em $C$, Números Complexos, Unidade de Aprendizagem, Matemática.

\section{INVESTIGATING IN $C$ : AN ONLINE UNIT OF LEARNING FOR STUDY OF COMPLEX NUMBERS}

Digital resources offer important opportunities to the process of teaching and learning of Mathematics, such as simulation, visualization, experimentation, among others. This way, it describes the development of the online learning unit "Investigating in $C$ ", directed to the study of Complex Numbers in Secondary School. The unit was developed in HTML and contains, among other features, 13 applets designed with the objective of enabling the study of Complex Numbers, in association with Analytical Geometry, and a pack of investigative activities related to the applets. It further examines the results of validation of the unit, performed by teachers and undergraduated students in Mathematics, using a mini-course. The results of the validation showed that the unit is adequate to the purpose of collaborating in the study of Complex Numbers, from a less algebraic perspective.

Keywords: Investigating in $C$, Complex Numbers, Learning Unit, Mathematics. 


\section{Introdução}

$\mathrm{Na}$ sociedade atual, na qual as transformações ocorrem rapidamente, é fundamental favorecer as condições para que o indivíduo construa seu conhecimento e desenvolva sua autonomia, senso crítico e responsabilidade. Nesse sentido, a Matemática ensinada nas escolas tem recebido algumas críticas. Segundo D’Ambrósio (2001), esta é ultrapassada, antiga e descontextualizada, o que causa desinteresse dos alunos. Moysés (2007) afirma que os temas matemáticos são, muitas vezes, trabalhados, na escola, de forma isolada do mundo que a rodeia.

As Tecnologias de Informação e Comunicação (TICs), utilizadas em atividades de investigação, podem ser recursos favoráveis para aprendizagem de Matemática, tanto no ensino a distância quanto de forma presencial. Como defendido por Ponte, Oliveira e Varandas (2003), as TICs permitem dar maior destaque ao papel da linguagem gráfica, relativizando a importância do cálculo e da manipulação simbólica. O enfoque gráfico favorece reflexões e análises críticas, possibilitando procedimentos menos mecânicos do que os, puramente, algébricos.

O presente artigo é resultado das atividades realizadas em um projeto de pesquisa ${ }^{1}$. No âmbito desse são desenvolvidos e disponibilizados, na Internet, recursos didáticos que possibilitam o estudo de temas matemáticos. $\mathrm{O}$ objetivo de tais ações é incentivar a utilização adequada das TICs em práticas pedagógicas, tendo em vista a melhoria do processo de ensino e aprendizagem de Matemática, no Ensino Médio.

Dentre os recursos desenvolvidos no âmbito do referido projeto, tem-se a unidade de aprendizagem online, "Investigando em $C$ " (http://www.es.cefetcampos.br/softmat/projeto_TIC/Investigando_em_C/), destinada ao estudo de Números Complexos, no Ensino Médio. Esta contém, entre outros recursos, 13 applets para o estudo de Números Complexos e uma apostila de atividades investigativas relacionadas aos applets. A unidade "Investigando em $C$ " pode, então, ser vista como um conjunto de objetos de aprendizagem ${ }^{2}$, por conter diversos applets, ou ela própria, em sentido amplo, pode ser vista como um objeto de aprendizagem.

Nesse contexto, este artigo descreve os objetivos, o desenvolvimento e os resultados da validação preliminar da unidade de aprendizagem online "Investigando em $C$ ". Para tanto, nas seções 2 e 3, descrevem-se, respectivamente, as motivações e as fases de desenvolvimento da referida unidade (concepção, preparação, implementação e validação). $\mathrm{Na}$ seção 4, analisam-se os resultados da validação, realizada com um grupo de licenciandos e professores de Matemática, por meio de um minicurso. Finalizando, a seção 5 apresenta algumas considerações sobre o trabalho desenvolvido.

\section{Desenvolvendo a Unidade "Investigando em $C$ ": panorama}

Números Complexos é um tema matemático presente em, praticamente, todos os grandes ramos da Matemática, tais como Álgebra, Topologia, Geometria (Analítica, Diferencial ou Algébrica), Análise, entre outros (Lima, 1985). Além disso, os Complexos estão presentes em aplicações como dinâmica dos fluidos e eletromagnetismo (Lima, 1985).

A importância desse tema matemático e as possibilidades que as TICs representam, em termos de recursos didáticos, motivaram o desenvolvimento de uma unidade de aprendizagem online para esse tema. Além disso, uma pesquisa mostrou que das 47 escolas de Ensino Médio existentes em 2004, no município de Campos dos Goytacazes (estaduais, municipais, federais e particulares), apenas 53\% trabalhavam o tema Números Complexos (Batista, 2004). Este resultado indica que muitos alunos terminaram o Ensino Médio sem estudar esse tema matemático, na referida cidade (na qual se localiza o IF Fluminense Campus Campos-Centro). Nesse sentido, a unidade de aprendizagem destinada ao estudo de Números Complexos poderá ser útil para alunos 
que não tiveram oportunidade de estudar este tema, mas que desejam fazê-lo, além de colaborar com estudo regular do mesmo.

A unidade de aprendizagem "Investigando em $C$ " é composta de páginas HTML e contém13 applets para estudo do tema Números Complexos. Applets (applets Java) são programas desenvolvidos em linguagem de programação Java ${ }^{\circledR}$, que podem ser incluídos em códigos HTML (Deitel H.; Deitel. P., 2003). Estes, em geral, visam a adicionar interatividade a aplicações Web. Os applets da unidade permitem o estudo de Números Complexos associado à Geometria Analítica, o que favorece uma análise menos algébrica do tema. A unidade contém, ainda, uma apostila de atividades investigativas associadas aos applets, aspectos históricos sobre Números Complexos e links para outros endereços que abordam o tema.

O GeoGebra (http://www.geogebra.at/) foi o software utilizado no desenvolvimento dos applets. Trata-se de um software gratuito, que possibilita o trabalho com Matemática Dinâmica. A expressão "Matemática Dinâmica" é utilizada por Markus Hohenwarter, criador do GeoGebra, ao explicar as funções do mesmo. Seria uma extensão da definição de "Geometria Dinâmica". Segundo Braviano e Rodrigues (2002), a Geometria Dinâmica permite a elaboração de construções eletrônicas, nas quais os elementos básicos podem ser movimentados na tela do computador sem alterar as posições relativas entre esses elementos e os objetos construídos a partir deles. Além de objetos geométricos, o GeoGebra dá um caráter dinâmico a outros objetos matemáticos como funções, gráficos, números, fórmulas, entre outros, o que justifica a expressão "Matemática Dinâmica".

Os applets da unidade "Investigando em $C$ " foram desenvolvidos como construções no GeoGebra e transformados em applets por meio de recursos deste próprio software. A facilidade de gerar os applets no GeoGebra foi um aspecto bastante favorável ao desenvolvimento destes recursos, fundamentais na composição da unidade.

O trabalho realizado foi motivado, também, por experiências anteriores, vivenciadas no âmbito do projeto de pesquisa, relacionando ações pedagógicas e uso das TICs.

\section{Material e Métodos: descrição das etapas de desenvolvimento}

O desenvolvimento da unidade de aprendizagem foi dividido em quatro etapas: concepção do projeto, planificação, implementação e validação (Behar, 2009). Na etapa de concepção, foram definidos o objetivo geral e o público alvo da unidade: colaborar para o estudo de Números Complexos, no Ensino Médio, por meio de recursos interativos. A unidade é direcionada a alunos do Ensino Médio, no entanto, poderá ser utilizada por alunos da Licenciatura em Matemática. Também na etapa de concepção, foi estabelecido que a proposta da unidade seria fundamentada na teoria sócio-histórica A mediação, nesta teoria, inclui o uso de instrumentos e de signos no contexto social, e a combinação de uso desses recursos mediadores possibilita o desenvolvimento dos processos psicológicos superiores (Vygotsky, 2007). Segundo Vygotsky (2007), o processo de ensino e aprendizagem deve se adiantar ao desenvolvimento, criando a Zona de Desenvolvimento Proximal (ZDP). Os recursos pedagógicos (applets e apostila de atividades), desenvolvidos e disponibilizados na unidade, são instrumentos mediadores que, bem utilizados, podem contribuir para a criação de ZDPs.

$\mathrm{Na}$ etapa de planificação, definiu-se a estrutura da unidade, estabelecendo que esta seria desenvolvida em HTML, contendo, principalmente, applets e uma apostila de atividades investigativas. Além disso, foram definidos os objetivos de dez dos treze applets (a necessidade dos outros três applets foi observada durante a fase de implementação, tendo em vista favorecer a sequência entre os tópicos abordados).

$\mathrm{Na}$ etapa de implementação foram definidos, inicialmente, os softwares a serem utilizados: i) GeoGebra (http://www.geogebra.org/cms/) na construção dos applets; ii) NVU (http://nvudev.com/index.php) no desenvolvimento das páginas HTML; iii) 
Régua e Compasso (http://www.ntegravatai.relrs.g12.br/progr/ReC/) e FX - The Efeito Generator (http://www.effectgenerator.com) na elaboração de animações; iv) Wink (http://www.debugmode.com/wink/) na elaboração de tutorial. A seguir, foram construídos os applets que compõem a unidade de aprendizagem, tendo sempre em vista que estes deveriam ser interativos e possibilitar o estabelecimento de conjecturas. Em paralelo às revisões finais dos applets, foi desenvolvida uma apostila de atividades investigativas. Estas atividades apresentam uma abordagem diferenciada dos exercícios tradicionais dos livros didáticos e devem ser realizadas utilizando os applets. São descritos, brevemente, a seguir, os objetivos de alguns applets, assim como, a estrutura da apostila de atividades.

- Plano Complexo: visa a apresentar o plano complexo ou de Argand-Gauss (Figura 1).

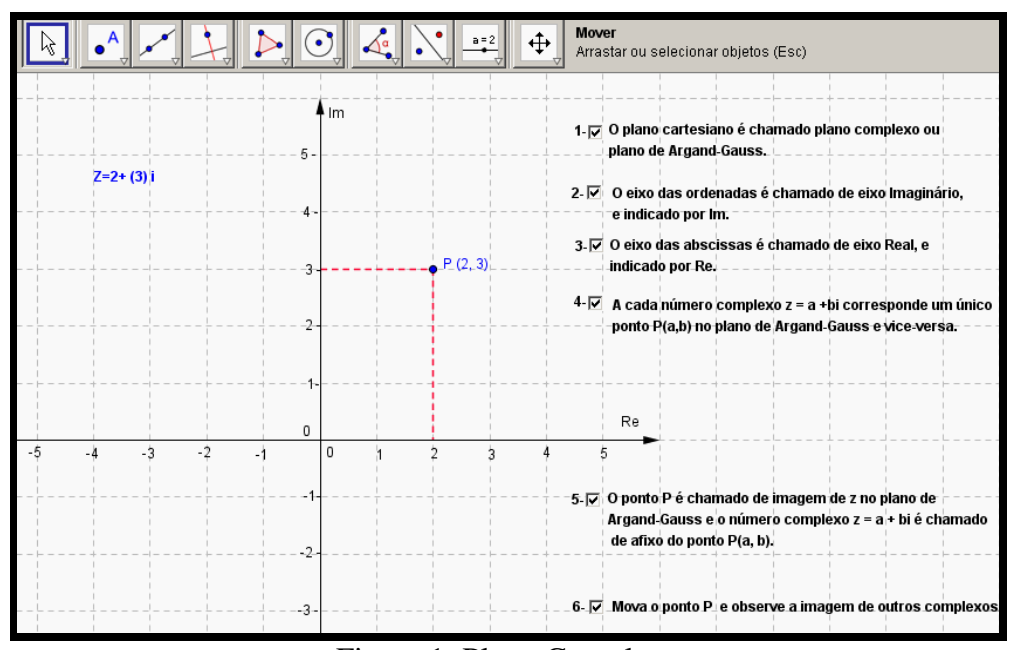

Figura 1: Plano Complexo

A figura 1 mostra o número complexo $\mathrm{z}=\mathrm{a}+\mathrm{bi}$ (parte superior esquerda), que corresponde ao ponto $\mathrm{P}(\mathrm{a}, \mathrm{b})$, marcado no plano complexo. $\mathrm{O}$ ponto $\mathrm{P}$ pode ser movimentado e os valores de $\mathrm{a}$ e $\mathrm{b}$, do número complexo $\mathrm{z}$, mudam de acordo com $\mathrm{a}$ posição do ponto $\mathrm{P}$ no plano. Os eixos das ordenadas e das abscissas são indicados por Im e Re, respectivamente. No applet, aparecem caixas numeradas que devem ser marcadas na seqüência sugerida. Esta ação faz aparecer na tela textos explicativos e/ou figuras sobre o tema em questão (em 11 dos 13 applets elaborados há essas caixas).

- Adição de Números Complexos: tem por objetivo possibilitar a visualização geométrica da soma de dois números complexos, considerando cada um deles como um vetor com origem no ponto $(0,0)$ (Figura 2$)$.

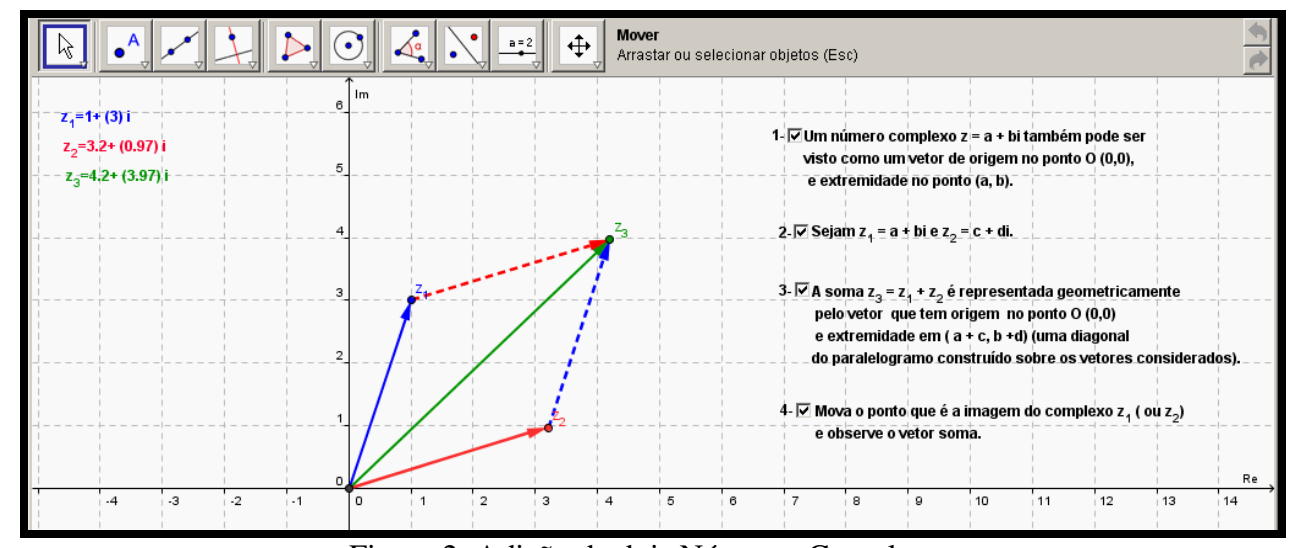

Figura 2: Adição de dois Números Complexos

Os números complexos, apresentados na parte superior esquerda do applet, estão representados, no plano complexo, por vetores com origem no ponto $(0,0)$. Para facilitar 
o entendimento, foram utilizadas cores iguais para escrever o número complexo e traçar o vetor que o representa. Os vetores que representam os complexos são sempre traçados em linha contínua. Os vetores em linha tracejada são paralelos ao de mesma cor, em linha contínua. A soma dos números complexos $\mathrm{z}_{1}$ e $\mathrm{z}_{2}$ é o complexo $\mathrm{z}_{3}$. Geometricamente, a referida soma é representada por um vetor de origem em $(0,0)$ e ponto final na imagem de $\mathrm{z}_{3}$, formando uma das diagonais do paralelogramo definido pelos vetores que representam $\mathrm{z}_{1}$ e $\mathrm{z}_{2}$.

- Potências de i: visa a mostrar, geometricamente, que os vetores que representam as potências $i^{\mathrm{n}}$ (n natural) são obtidos por rotações de $90^{\circ}$, em relação à origem $(0,0)$, do vetor que representa a potencia $i^{0}$, no sentido anti-horário. São representadas as potências de $\mathrm{i}$, por meio da rotação dos vetores, a fim de que o usuário possa identificar a repetição a partir de $i^{4}$ (Figura 3).

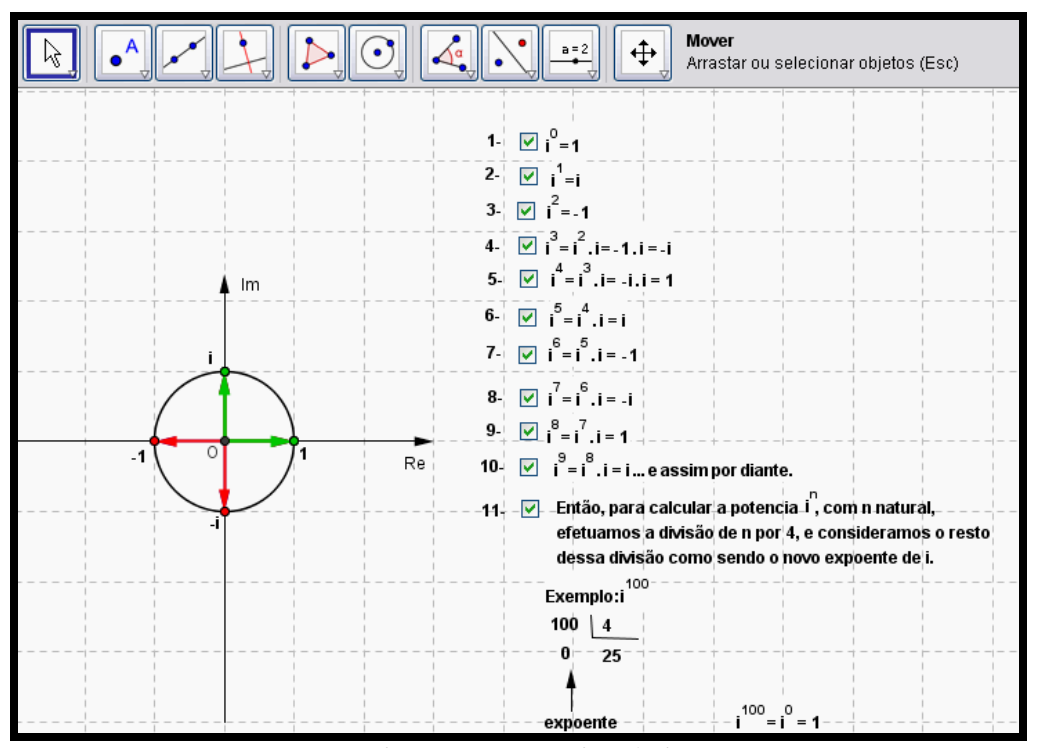

Figura 3: Potências de i

- Multiplicação por Unidade Imaginária: visa a mostrar, geometricamente, que ao multiplicar um número complexo $\mathrm{z}_{1}$ por $\mathrm{i}$, o vetor que representa $\mathrm{z}_{1}$ sofre uma rotação de $90^{\circ}$, em relação à origem $(0,0)$, no sentido anti-horário (Figura 4).

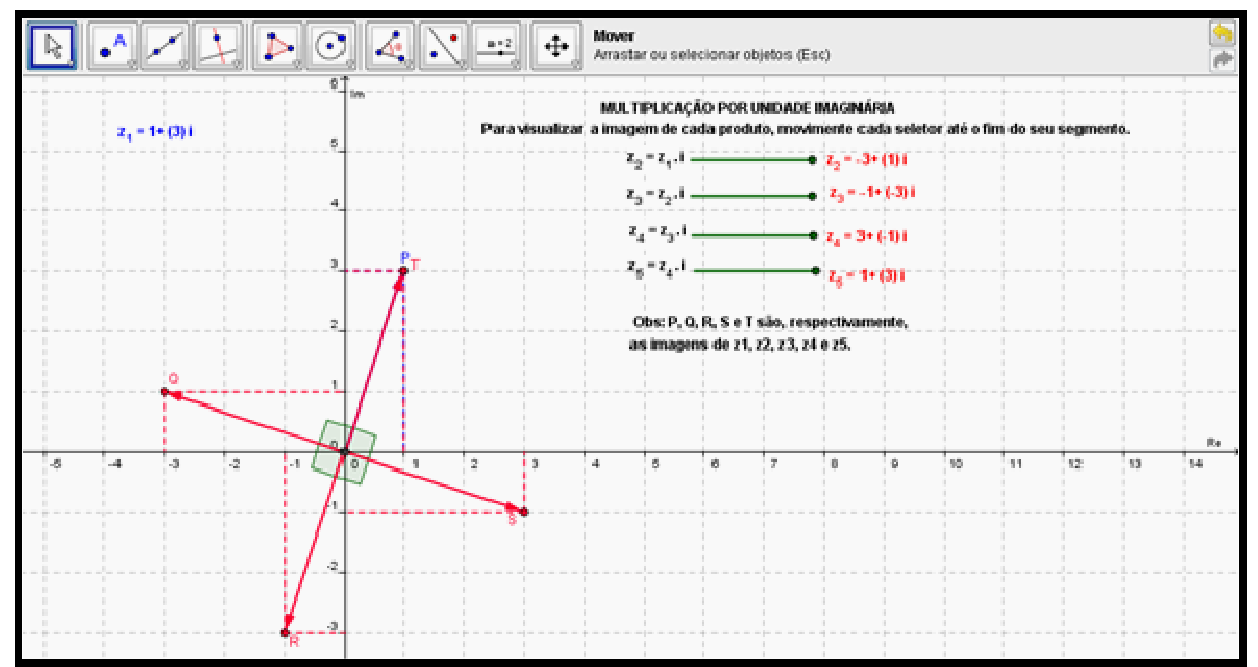

Figura 4: Multiplicação por Unidade Imaginária

A movimentação dos seletores promove uma rotação do vetor correspondente a $\mathrm{z}_{1}$, no sentido anti-horário, de $90^{\circ}, 180^{\circ}, 270^{\circ}$ e $360^{\circ}$, respectivamente, quando o número complexo $\mathrm{z}_{1}$ é multiplicado por $\mathrm{i}, \mathrm{i}^{2}, \mathrm{i}^{3}, \mathrm{i}^{4}$. 
Os applets são instrumentos mediadores que possibilitam movimentações e, assim, contribuem para o estabelecimento de conjecturas. Dessa forma, estes recursos permitem que as reflexões, realizadas com seu auxílio, adquiram características qualitativamente positivas, o que contribui para o desenvolvimento dos processos psicológicos superiores.

Assim como os applets, as atividades da apostila são instrumentos mediadores. A referida apostila contém 13 atividades investigativas, as quais visam levar o aluno a refletir sobre os resultados obtidos por meio da manipulação dos applets. O quadro 1 mostra um exemplo desse tipo de atividade. A apostila contém, ainda, uma segunda parte com 16 exercícios de aplicação dos conceitos abordados nas referidas atividades investigativas.

Quadro 1: Exemplo de Atividade Investigativa

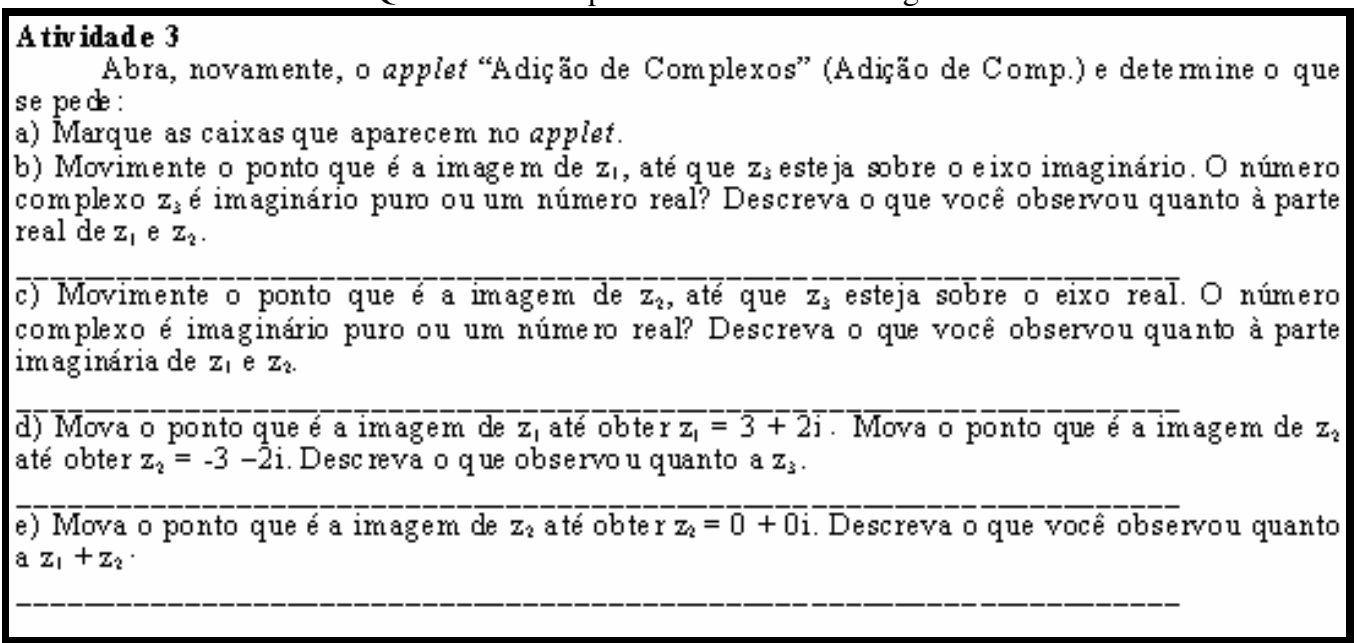

Após a elaboração e várias revisões desses recursos didáticos, teve início o desenvolvimento da unidade de aprendizagem propriamente dita. Essa foi desenvolvida em HTML, contendo os recursos mencionados, além de aspectos históricos sobre Números Complexos e links para outros endereços que abordam este tema matemático.

Descrevem-se, a seguir, as seções da unidade "Investigando em C". A Figura 5 mostra a tela de introdução da unidade. A imagem que aparece nessa tela não é estática, trata-se de uma animação que mostra os vetores que representam todos os números complexos que possuem módulo (comprimento) 1.

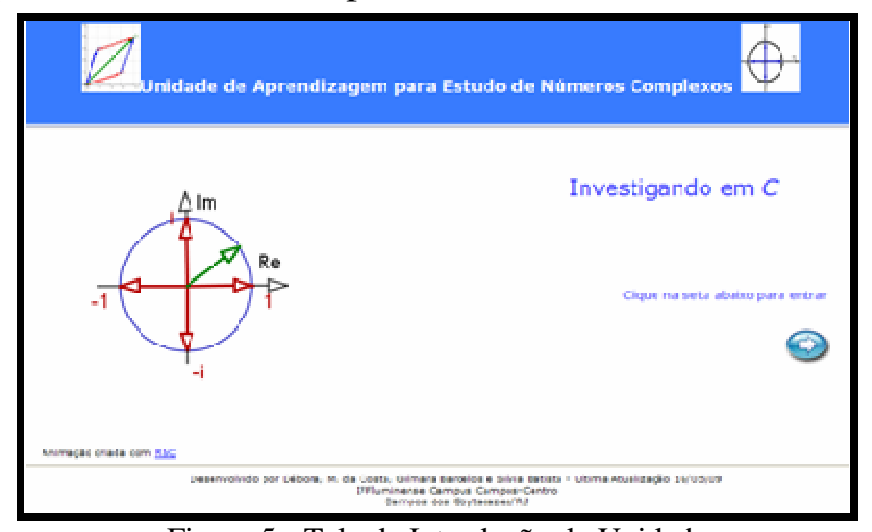

Figura 5 - Tela de Introdução da Unidade

A figura 6 mostra a tela de apresentação da unidade. Na lateral direita encontra-se o menu principal, que permite acesso às seções da unidade. Além da seção Apresentação, a unidade possui outras cinco seções: i) Aspectos Históricos, na qual se descreve um pouco da história dos Números Complexos; ii) Applets, na qual é possível acessar 
qualquer um dos 13 applets; iii) Apostila, na qual a apostila de atividades é disponibilizada; iv) Links, na qual é possível acessar outros endereços eletrônicos relacionados ao tema Números Complexos; v) Créditos, na qual são apresentados os nomes dos autores e dos softwares utilizados.

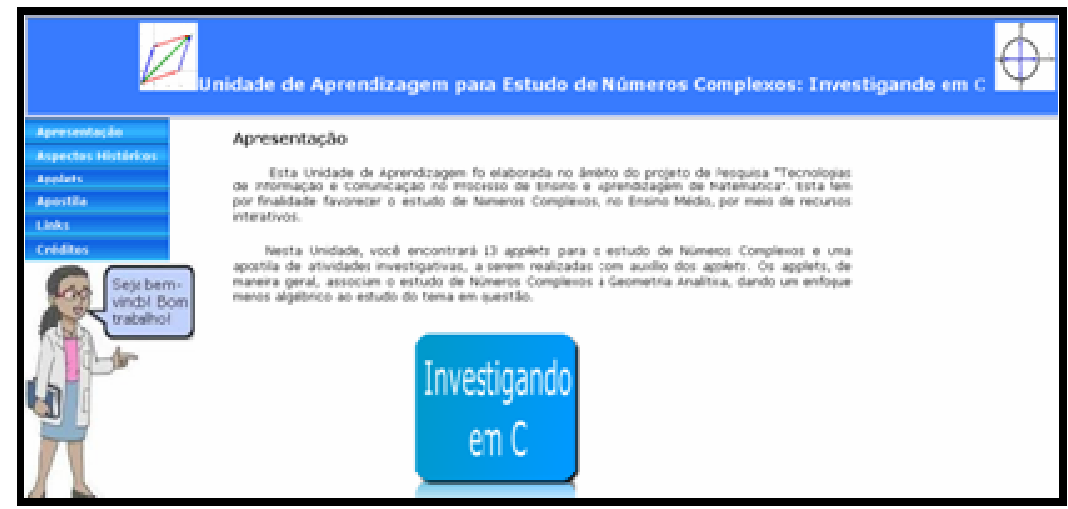

Figura 6 - Apresentação da Unidade

$\mathrm{Na}$ quarta etapa, a unidade "Investigando em C" passou por uma validação preliminar (validação em forma de estudo piloto) com licenciandos e professores de Matemática, objetivando identificar possíveis falhas. A referida validação ocorreu por meio de um minicurso, ministrado pelas autoras deste artigo, no IF Fluminense Campus Campos-Centro, com sete participantes. O minicurso foi realizado em maio de 2009, com duração de quatro horas (divididas em dois encontros). Esse tipo de validação é fundamental para a identificação de pontos a serem melhorados, sob o ponto de vista de quem já conhece o conteúdo. Destaca-se que a validação in loco, com alunos do Ensino Médio, está prevista para ocorrer no segundo semestre de 2009 e permitirá analisar a adequação da unidade ao seu público alvo.

Todas as etapas de desenvolvimento da unidade foram norteadas pela experiência de suas desenvolvedoras, tanto em termos de desenvolvimento de recursos didáticos, quanto em relação à experiência pedagógica com Matemática do Ensino Médio.

\section{Resultados e Discussão}

Descrevem-se, nesta seção, a coleta e análise de dados da validação preliminar.

Iniciando o primeiro encontro, as seções da unidade de aprendizagem foram visitadas pelos participantes, sob a orientação das mediadoras. A seguir, foi realizada uma breve descrição das ferramentas disponíveis nos applets. Os participantes resolveram, então, as atividades 1, 2 e 3 da apostila, utilizando os applets correspondentes. Ao final do primeiro encontro, foi realizada a socialização e formalização das conjecturas estabelecidas nas referidas atividades.

No segundo encontro, foram resolvidas as outras 10 atividades, utilizando os applets correspondentes, ocorrendo, ao final, a socialização e formalização das conjecturas. A segunda parte da apostila não foi resolvida durante a validação, porém esse fato não prejudicou a validação, uma vez que os exercícios dessa parte são semelhantes aos que os livros didáticos apresentam, ou seja, já conhecidos dos participantes do minicurso. Encerrou-se esse encontro com os sete participantes respondendo a um questionário, o qual continha 11 perguntas (duas fechadas, sete semiabertas e duas abertas) sobre os applets e as atividades, assim como, sobre a unidade de aprendizagem propriamente dita.

A observação das atitudes, dos questionamentos e da resolução das atividades durante os dois encontros possibilitaram o registro de situações importantes para o alcance dos objetivos da validação. Os participantes utilizaram a unidade, resolveram as atividades e manipularam os applets com facilidade. Durante a realização da validação preliminar, não foi observada a necessidade de alteração nas páginas da unidade, nem 
nos applets. No entanto, foi possível observar que o item $g$ da atividade 1 , que solicita que as construções feitas no applet sejam salvas, deveria conter orientações para realizar tal procedimento, posto que alguns participantes apresentaram dúvidas em relação ao mesmo.

Após a realização do minicurso, foi promovida a análise dos dados levantados por meio do questionário. As respostas consideradas mais significativas são comentadas a seguir.

Quando questionados sobre a possibilidade de aplicar parte do minicurso na prática docente, todos os participantes responderam positivamente. Destacam-se os comentários registrados por dois alunos:

"Tanto a visualização quanto a movimentação dos applets são fáceis para o aluno entender" (Participante 1).

"Porque facilita a compreensão e ajuda a entender a parte algébrica" (Participante 4).

Com relação às atividades, todos afirmaram que as mesmas contribuem para a compreensão do tema em estudo e consideraram os enunciados claros. Em geral, isto foi justificado pela importância da representação geométrica, que permite melhor entendimento do assunto. O nível das atividades foi considerado "moderado" por 57\% dos participantes e "fácil" pelos demais. Embora o tema em estudo faça parte da matriz curricular do Ensino Médio, a resolução de atividades investigativas não é muito comum, o que justifica o fato de mais de $50 \%$ dos participantes terem considerado o nível das atividades como "moderado".

Todos os participantes consideraram que o uso de applets favorece a construção de conhecimentos matemáticos. Esse fato reforça a importância do uso de instrumentos no desenvolvimento do indivíduo, como defende a teoria sócio-histórica. Destacam-se algumas justificativas dadas pelos participantes:

"Pela visualização (congruências e semelhanças), você vendo fica muito mais convencido" (Participante 5).

"Pois o aluno consegue manipular e visualizar, construindo o conhecimento" (Participante 1).

A visão desses participantes está coerente com a de Santos (2008), quando este afirma que, por meio dos applets, os alunos podem interagir com o conteúdo (experimentação), criando uma intuição sobre o conceito explorado, construindo-o de forma mais consistente.

Questionados sobre a utilização de recursos tecnológicos digitais em aulas que ministraram (mesmo durante estágios), aproximadamente $86 \%$ dos participantes responderam já o terem feito e ressaltaram que as dificuldades são compensadas pelos resultados obtidos. Os participantes afirmaram, ainda, que os jovens se interessam por tecnologias e que estas tornam a aula mais dinâmica.

Quanto aos critérios relacionados à usabilidade, a unidade de aprendizagem foi muito bem avaliada, conforme mostra o gráfico 1. A usabilidade da interface de uma unidade de aprendizagem online é um aspecto que requer cuidado, pois esta, se bem planejada, aumenta as chances dos objetivos serem atingidos. Os resultados mostrados no gráfico 1 refletem a preocupação em desenvolver uma unidade fácil de usar, coerente em seu design (com uniformidade na estrutura das páginas), simples de navegar e com recursos interativos que possibilitam a reflexão e a construção de conhecimentos.

A última pergunta solicitava sugestões e/ou críticas. Um participante sugeriu fazer uso da unidade de aprendizagem (enfoque geométrico), paralelamente, à abordagem que os livros didáticos apresentam (abordagem algébrica). Outro sugeriu que a abordagem geométrica deveria ser feita após a abordagem algébrica. Vale ressaltar que ambos consideraram importante o uso da unidade, diferindo apenas na ordem do referido uso. Os demais participantes não deram sugestões. 

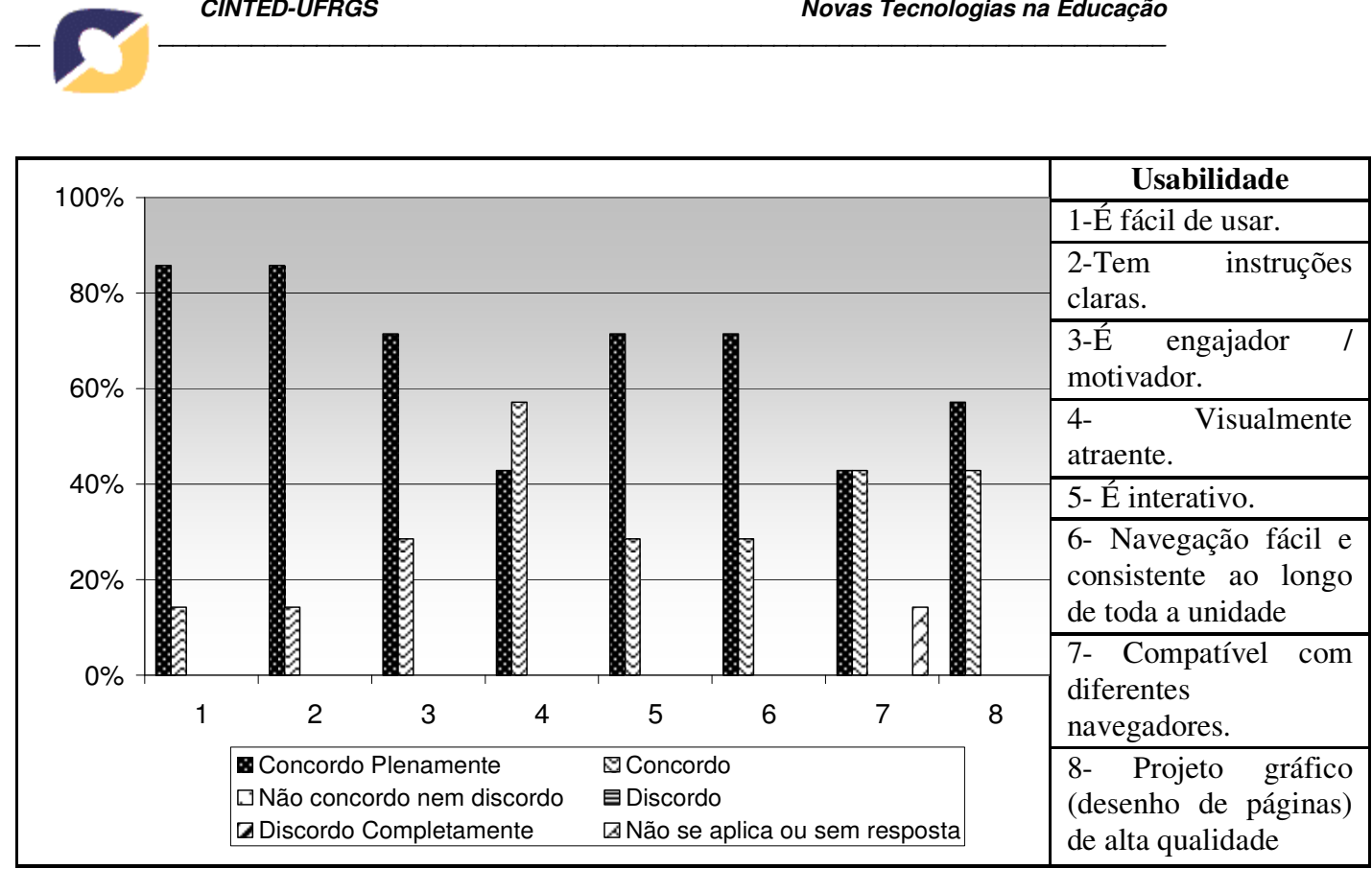

Gráfico 1: Avaliação da Unidade quanto à Critérios de Usabilidade

De maneira geral, a observação das ações dos participantes e a análise dos dados levantados pelos questionários mostraram que a unidade "Investigando em $C$ " e os recursos disponibilizados na mesma estão adequados aos seus objetivos.

\section{Considerações Finais}

Com a unidade "Investigando em $C$ " espera-se, como já mencionado, colaborar para o processo e ensino e aprendizagem de Números Complexos, no Ensino Médio. A referida unidade foi desenvolvida sob um enfoque sócio-interacionista, assim os applets e as atividades propostas, que não são convencionais como as, em geral, apresentadas nos livros didáticos, são vistos como instrumentos mediadores. O uso desses instrumentos, segundo Vygotsky (2007), reorganiza, de forma radical, as funções psicológicas superiores, tais como memória e atenção.

A realização da validação preliminar foi muito importante. Os participantes mostraram muito interesse durante o minicurso, o que pode ser atribuído à qualidade dos recursos e ao fato do enfoque, dado ao tema, ser diferente do que eles conheciam. As atitudes dos participantes, seus questionamentos e as respostas dos questionários indicaram que a unidade de aprendizagem está coerente com seus objetivos.

Destaca-se que a unidade será complementada com applets para o estudo de potenciação e radiciação de números complexos e com uma seção contendo aplicações práticas do referido tema em outras áreas de conhecimento. É importante que a unidade de aprendizagem esteja em constante processo de desenvolvimento, pois atualizações e ampliações vão enriquecendo-a. Como mencionado, também está prevista a validação da unidade com alunos de Ensino Médio, pois é fundamental testar o recurso, diretamente, com seu público alvo. A partir da referida validação, a unidade será aperfeiçoada, considerando as necessidades e o feedback dos alunos do Ensino Médio.

Por fim, ressalta-se o que desenvolvimento de recursos digitais para educação é um processo muito rico, permeado por algumas inquietações decorrentes de problemas relacionados à tecnologia e/ou ao tema de estudo específico. Como a abordagem do assunto não é a tradicional, o desenvolvimento destes recursos requer uma fundamentação teórica consistente que permita propor novas estratégias para o tema em questão. Com relação à tecnologia, nem sempre é possível obter, exatamente, o que se deseja e, assim, soluções devem ser discutidas em busca do melhor caminho.

\section{Notas de Texto}


1 Projeto "Tecnologias de Informação e Comunicação no Processo de Ensino e Aprendizagem de Matemática", vinculado ao IF Fluminense Campus Campos-Centro.

${ }^{2}$ Um objeto de aprendizagem pode ser entendido como "qualquer recurso digital que possa ser reutilizado para dar suporte ao ensino" (Wiley, 2001, p. 7). Qualquer material eletrônico que contenha informações que possibilitem a construção do conhecimento pode ser considerado um OA, seja essa informação em forma de imagem, página HTML, animação ou simulação (RIVED, s.d).

\section{Referências Bibliográficas}

BATISTA, S. C. F. SoftMat: Um Repositório de Softwares para Matemática do Ensino Médio - Um Instrumento em Prol de Posturas mais Conscientes na Seleção de Softwares Educacionais. Dissertação de Mestrado em Ciências de Engenharia. Campos dos Goytacazes, RJ, Universidade Estadual do Norte Fluminense - UENF, 2004.

BEHAR, P. e Colaboradores. Modelos Pedagógicos em Educação a Distância. ArtMed: Porto Alegre, 2009.

BRAVIANO, R.; RODRIGUES, M. H. W. L. Geometria Dinâmica: Uma nova Geometria. Revista do Professor de Matemática, São Paulo: Sociedade Brasileira de Matemática, n. 49, p. 22-26, 2002.

D'AMBRÓSIO, U. Educação Matemática: da Teoria à Prática. 8. ed. Campinas, SP: Papirus, 2001.

DEITEL, H. M; DEITEL, P. J. Java, como programar. Tradução de Carlos Arthur Lang Lisboa. 4. ed. Porto Alegre: Bookman, 2003.

LIMA, E. L. Sobre a Evolução de Algumas Idéias Matemáticas. Revista do Professor de Matemática (RPM). n. 06, p. 1-8. São Paulo: Sociedade Brasileira de Matemática, 1985.

MOYSÉS, L. Aplicações de Vygotsky à Educação Matemática. 8.ed. Campinas-SP: Papirus, 2007.

PONTE, J. P.; OLIVEIRA, H.; VARANDAS, J. M. O Contributo das Tecnologias de Informação e Comunicação para o Desenvolvimento do Conhecimento e da Identidade Profissional. In: FIORENTINI, D. (Ed.). Formação de professores de Matemática: Explorando novos caminhos com outros olhares. Campinas: Mercado de Letras, 2003. p. 159-192.

RIVED. Conheça o RIVED. s.d. Disponível em: $<$ http://www.rived.mec.gov.br/site_objeto_lis.php>. Acesso em: 10/04/09.

SANTOS, V. C. P. Mathlets: Possibilidades e Potencialidades para uma Abordagem Dinâmica e Questionadora no Ensino de Matemática. Dissertação de Mestrado em Ensino de Matemática. Rio de Janeiro, RJ. Universidade Federal do Rio de Janeiro UFRJ, 2008.

VYGOTSKY, L. S. A formação social da mente: o desenvolvimento dos processos psicológicos superiores. 7.ed. São Paulo, Martins Fontes, 2007.

WILEY, D. Connecting learning objects to instrucional design theory: a definition, a metaphor, and taxonomy. 2001. Disponível em: <www.reusability.org/read/chapters/wiley.doc>. Acesso em: 10/04/09. 